\title{
The triticeous cartilage - redefining of morphology, prevalence and function
}

\author{
A. Vatansever ${ }^{1}$, D. Demiryürek², i. Tatar², B. Özgen ${ }^{3}$ \\ ${ }^{1}$ Department of Anatomy, Faculty of Medicine, Balıkesir University, Balikesir, Turkey. \\ 2Department of Anatomy, Faculty of Medicine, Hacettepe University, Ankara, Turkey \\ ${ }^{3}$ Department of Radiology, Faculty of Medicine, Hacettepe University, Ankara, Turkey
}

[Received: 23 January 2018; Accepted: 7 March 2018]

Background: Triticeous cartilage is a small cartilaginous component of the laryngeal skeleton. This cartilage, located in posterior end of the thyrohyoid ligament, presents in different shapes. Radiological studies indicate clinical and anatomical importance of the triticeous cartilage but these studies have limited information due to inadequate inspection method. Computed tomographic angiography is able to evaluate the triticeous cartilage with using three-dimensional images in more detail. The aim of this study is to describe prevalence and morphological properties of the triticeous cartilage.

Materials and methods: We examined computed tomographic angiography images of 746 patients (368 women, 378 men) retrospectively. Shapes, calcification degrees, volumes, lengths and wideness of the triticeous cartilage were evaluated by OsiriX-Lite software.

Results: According to our results, triticeous cartilage presents common in the examined population (68.1\%). The prevalence of the triticeous cartilage was higher in men than in women. We also found that the degree of calcification was not related with age and gender.

Conclusions: Clinical importance of the triticeous cartilage is that it could be misdiagnosed with atherosclerosis in common carotid artery because the triticeous cartilage is located almost at same level as the bifurcation of the common carotid artery. Therefore, clinicians should be aware about the triticeous cartilage. (Folia Morphol 2018; 77, 4: 758-763)

Key words: atherosclerosis, calcification, computed tomographic angiography, larynx

\section{INTRODUCTION}

Triticeous cartilage (TC) is a small cartilaginous component of the laryngeal skeleton. It may be found within the lateral thyrohyoid ligament which extends between the superior horn of the thyroid cartilage and the greater horn of the hyoid bone. Embryologically, laryngeal cartilages develop from the fourth and the sixth pharyngeal arches and the development begins at around the $12^{\text {th }}$ week of gestation. However, the TC is not a constant structure, could be seen unilateral, bilateral or be absent, but once it is present, it does not disappear with age [18, 22]. Although calcification patterns of the laryngeal cartilages are not yet clear, Claassen et al. [6] hypothesized that the contents of extracellular matrix of the laryngeal cartilage might have a role during calcification processes. There is no known function of the TC but it is assumed that it supports the lateral thyrohyoid ligament $[3,18]$. Besides this, a cadaveric study demonstrated that one of the functions of TC could be muscle attachment [16].

Address for correspondence: Dr. A. Vatansever, Balikesir University, Faculty of Medicine, Department of Anatomy, 10145, Balikesir, Turkey, tel: +90 5383681312, fax: +90 2666121417, e-mail: alpervatansever@yahoo.com 


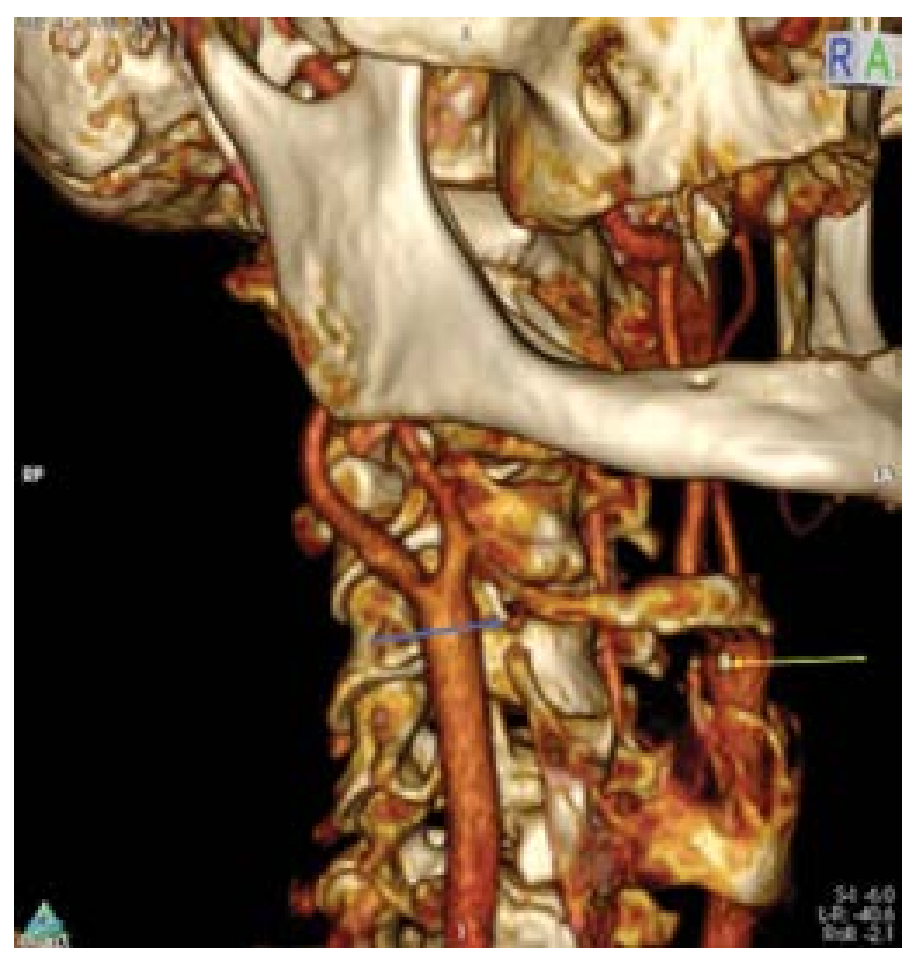

Figure 1. Three-dimensional reconstruction of computed tomographic angiography images. Blue arrow demonstrates triticeous cartilage, yellow arrow demonstrates atherosclerotic plaque in common carotid artery.

A number of studies reveal the importance of the presence of TC. These studies emphasized that the TC should not be confused with the atherosclerosis to be shaped in the common carotid artery as is shown in Figure 1 [5, 7, 13, 15, 19]. However, these studies were completed by using $\mathrm{X}$-ray images and therefore they were unable to evaluate morphology of the TC. Additionally, the TC should be calcified in order to be observed in X-ray images. There are limited studies describing the calcification of the TC and differentiating this calcification from the atherosclerosis located in common carotid artery as shown in Figure $1[1,3,5]$. The TC could also be misdiagnosed as the fractures of the superior horn of the thyroid cartilage in forensic medicine [9]. They also mentioned that the TC could only be seen in X-ray images when it is ossified. Although there was limited description for the degree of calcification, comparison between calcification, age and gender has not been reported before [3]. Alqahtani et al. [3] have described scales of shape and calcification degrees of the TC and it was the first report about morphological classification of the TC. Hence, their study can be regarded as the basis for the morphological properties of the TC.

Dental studies have demonstrated that the TC could be seen in lateral and panoramic radiographic images at the level of the third and fourth cervical vertebrae $[1,5]$. It is important to recognise and differentiate the TC from pathologies located in common carotid artery. The disadvantage of the radiographic methods during the period of diagnosis is that the TC can only be seen when it is calcified in X-ray images.

Neck traumas and atherosclerosis are frequently seen and a number of patients suffering from these conditions report to hospitals. The aim of our study was to determine the prevalence of the TC in the population and to establish its volume, length, width, calcification percentage and density, hence, improving the anatomical knowledge of the laryngeal skeleton. In the present study, morphological properties of the TC in relation to gender and age group have been reported in the population for the first time.

Besides cadaveric studies, radiological studies are also important for clinical anatomy. Knowing and being aware about the radiological anatomy of the neck region is crucial for both medical students and clinicians.

\section{MATERIALS AND METHODS}

In this study, we retrospectively evaluated computed tomographic angiography (CTA) images of 746 patients ( 378 men and 368 women) who visited our 
Table 1. Prevalence of the triticeous cartilage (TC)

\begin{tabular}{llccc}
\hline & & \multicolumn{2}{c}{ Sex } & Total \\
\cline { 3 - 4 } & & Men & Women & \\
\hline TC & None & $88(23.3 \%)$ & $150(40.8 \%)$ & $238(31.9 \%)$ \\
& Unilateral & $86(22.8 \%)$ & $81(22.0 \%)$ & $167(22.4 \%)$ \\
& Bilateral & $204(54.0 \%)$ & $137(37.2 \%)$ & $341(45.7 \%)$ \\
& & $\mathbf{3 7 8 ( 1 0 0 \% )}$ & $\mathbf{3 6 8 ( 1 0 0 \% )}$ & $\mathbf{7 4 6 ( 1 0 0 \% )}$ \\
\hline
\end{tabular}

University Hospital for various reasons. The range of the age was between 25 and 111. All patients were examined on a Somatom Definition (Siemens, Erlangen, Germany) and Sansation 16 (Siemens, Germany) computed tomography scanners. Patients underwent routine CTA protocols of the University Hospital.

All images were evaluated with OsiriX-Lite software after three-dimensional (3D)-reconstruction by a radiology specialist in the Radiology Department. Image analysis was carried out on bone and soft tissue windows. After determination of the thyrohyoid ligament, the cartilaginous or ossified structure was closely inspected throughout the entire length of the ligament within the same images. Data were collected from axial and sagittal CTA images. Length and width of the TC were evaluated from sagittal CTA images, while volume and density values were from axial sections. After observation of the thyroid cartilage and great horn of hyoid bone, we identified the TC in sagittal sections. Then, for length and width measurements, we used the best view of the TC. For volume measurement, the TC was monitored from the proximal to the distal in axial sections and region of interest $(\mathrm{ROI})$ for the $\mathrm{TC}$ was determined in each image that the TC was seen. Once the ROI selection was completed, the volume of the TC was calculated via OsiriX-Lite software. All authors confirmed that categorising of the shape and degree of the calcification was completed as described by previous study [3].

To determine the density, the image in which the best view of the TC could be seen in axial sections was selected. Then, the ROI was identified and the density of the TC was calculated via OsiriX-Lite software.

\section{Statistical analysis}

Statistical analyses were performed by using SPSS v21 software produced by IBM Knowledge Centre. For comparing length, width and volume of the TC between genders, we used a Mann-Whitney $U$ test at a significance level of 0.05 . We also performed comparisons between calcification, gender and age using bivariate correlation test at significance level 0.05 .
The study was started after the approval from University's Ethical Board (decree no. GO 15/599-06).

\section{RESULTS}

Table 1 shows the prevalence and distribution of the TC in both genders. According to our results, the TC is widely seen in the population. We demonstrated that $68.1 \%$ of the patients observed had at least one TC $(n=508)$ and the TC was most commonly seen bilaterally among these patients $(n=341)$.

Figures 2 and 3 show calcification degrees and appearances of the TC, respectively. We identified that cartilaginous TC was the most common degree of calcification (29.3\%). The less-common calcification degree was moderate triticeous calcification found in $17 \%$ (Table 2).

Table 3 shows the distribution of the appearance of the TC. The appearance classification of the TC was evaluated based on previous study [3]. While oval-shaped TC was the most frequently observed TC in the population (42.5\%), the teardrop-shaped TC was the least common type (2.8\%).

Length of the TC varied between $1.54 \mathrm{~mm}$ and $22.2 \mathrm{~mm}$ in sagittal sections of CTA images. Volume of the TC varied between $3.7 \mathrm{~mm}^{3}$ and $389 \mathrm{~mm}^{3}$. It was seen that as the length increased, the volume increased as well, and this was statistically significant ( $p<0.01$ ). Width of the TC was seen at between 1.34 $\mathrm{mm}$ and $6.07 \mathrm{~mm}$. Our data show that men have more common and bigger TCs than women and these results are statistically significant $(p<0.05)$ (Table 4).

As a result of evaluating the degree of calcification, it was determined that the degree of calcification was not related to age and gender ( $p<0.05)$. This result is similar to previous study [3].

\section{DISCUSSION}

Neck trauma is important due to regional structures, great vessels and nerves, respiratory and digestion pathways. Additionally, routine CTA procedures are performed to diagnose any vascular 


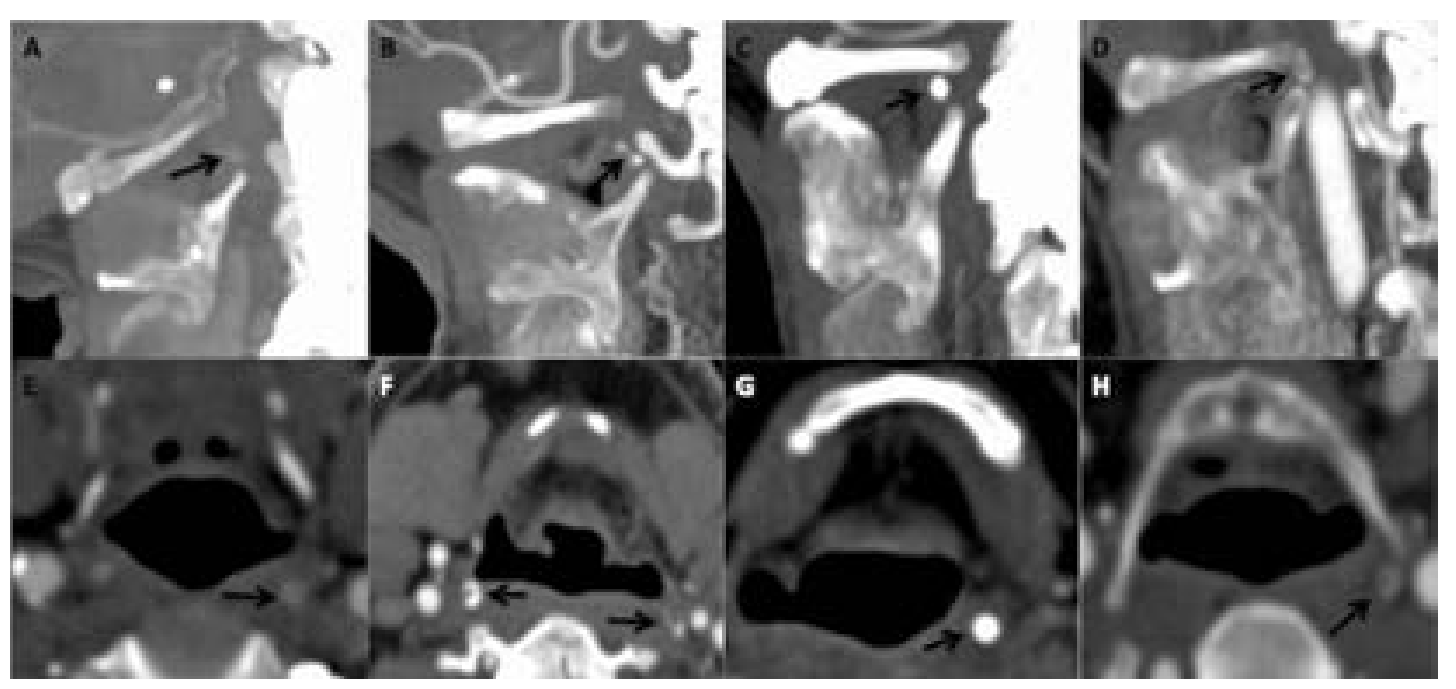

Figure 2. Calcification degree of the triticeous cartilage. A. Sagittal view of cartilaginous triticeous; B. Sagittal view of mild triticeous calcification; C. Sagittal view of moderate triticeous calcification; D. Sagittal view of marked triticeous calcification; E. Axial view of cartilaginous triticeous; F. Axial view of mild triticeous calcification; G. Axial view of moderate triticeous calcification; H. Axial view of marked triticeous calcification.

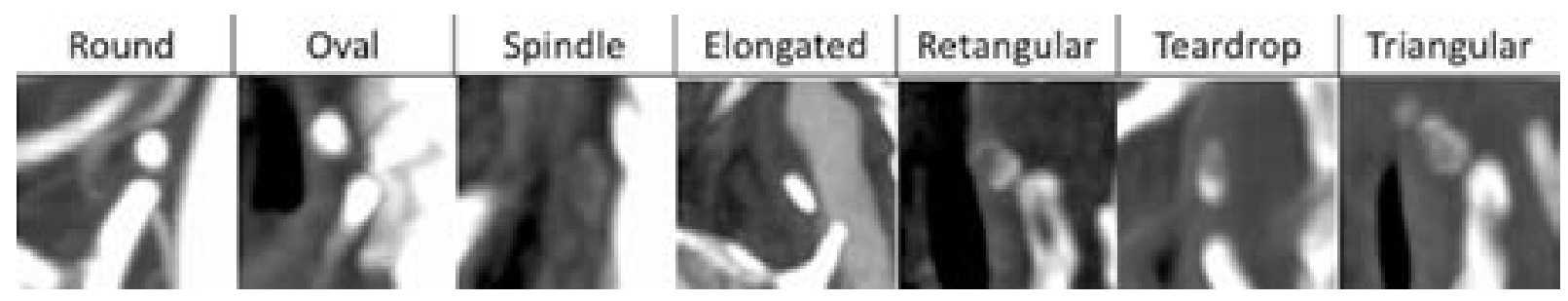

Figure 3. Appearance of the triticeous cartilage in sagittal computed tomographic angiography images.

Table 2. Distribution of calcification degree of the triticeous cartilage in Turkish population

\begin{tabular}{lc}
\hline Parameters & N (\%) \\
\hline Cartilaginous & $249(29.3 \%)$ \\
Mild & $144(17.0 \%)$ \\
Moderate & $227(26.7 \%)$ \\
Marked & $229(27.0 \%)$ \\
Total & $849(100.0 \%)$ \\
\hline
\end{tabular}

Table 3. Distribution of shape of the triticeous cartilage in Turkish population

\begin{tabular}{lc}
\hline Parameters & N (\%) \\
\hline Round & $76(9.0 \%)$ \\
Oval & $361(42.5 \%)$ \\
Spindle & $69(8.1 \%)$ \\
Elongated & $131(15.4 \%)$ \\
Rectangular & $160(18.8 \%)$ \\
Teardrop & $24(2.8 \%)$ \\
Triangular & $28(3.3 \%)$ \\
Total & $849(100.0 \%)$ \\
\hline
\end{tabular}

diseases at the early stages. Besides that, panoramic radiography is also an important imaging method for dentists and is used very frequently in clinics. Therefore, clinicians should be more careful and aware about the presence of TC for accurate diagnosis.

As is well known about of the anatomical organisation of the neck region, the common carotid artery ascends from the posterolateral side of the larynx. Therefore, atherosclerotic plaques forming in the carotid artery located more laterally than the TC and it is important while creating differential diagnosis list. Besides these, improving the anatomical knowledge of the larynx is crucial for anatomy education. As our results show, the TC can occur in various appearances and calcification degrees. Our study is the first report to identify the prevalence and the most detailed morphological properties of the TC among a large adult population.

In the last decade, many authors have studied the calcification of laryngeal cartilages. The thyroid cartilage is the one of the most interested one for 
Table 4. Mean diameters of the triticeous cartilage

\begin{tabular}{lcc}
\hline & Men & Women \\
\hline Volume $\left[\mathrm{mm}^{3}\right]$ & 46.72 & 23.94 \\
Length $[\mathrm{mm}]$ & 5.35 & 4.09 \\
Width $[\mathrm{mm}]$ & 3.42 & 2.85 \\
\hline
\end{tabular}

Table 5. Prevalence of the triticeous cartilage reported in different studies

\begin{tabular}{lc}
\hline & Percentage (\%) \\
\hline Vatansever et al. (2017) & 68.1 \\
Alqahtani et al. (2016) & 53.1 \\
Wilson et al. (2017) & 33 \\
Watanabe et al. (1982) & 65.5 \\
Ajmani et al. (1990) & 13 \\
Joshi et al. (2014) & 58 \\
\hline
\end{tabular}

recent studies $[8,11,14,17,20]$. Although there is no current consensus about age-related calcification yet, they still assume that age is one of most important factors for calcification of laryngeal skeleton. Our results showed that the age was not responsible for the calcification of the TC. But there is still very limited information about the TC.

Even though there are several studies about the determination of prevalence and morphology of the TC, only Alqahtani et al. [3] have studied the TC by using CTA images. They completed their study with 663 patients. According to their results, prevalence of the TC was $53.1 \%$, which was less than our results $(68.1 \%)$ (Table 5). Similar to their study, our results indicate that the TC was seen more commonly in men than in women. In contrast, our results showed that the percentage of cartilaginous form of the TC calcification was the highest in the population. This difference might be related with the life style, environment or race.

Additionally, we also evaluated distribution of shape of the TC in the population (Table 3). Oval-shaped TC was seen more common according to our results. When we compared degree of calcification and genders, there was no significant difference between them. The relation between density and the degree of calcification of the TC was statistically significant, but the density is not as progressive as calcification. To our knowledge of the literature, this is the first report that demonstrates comparison of density and degree of calcification of the TC.

As is well documented in panoramic radiography studies, the TC would easily be confused with the atherosclerosis formed in common carotid artery $[1,5,12-14,19]$. Additionally, as the TC may be found asymptomatic, it may also cause symptoms such as dysphagia and neck pain [4]. However, the TC can only be seen in X-ray images when it was calcified. Hence, $X$-ray studies provide limited information about the TC.

In a cadaveric study, prevalence of the TC was $33 \%$ in 87 adult cadavers [22]. In contrast to their results, our study showed that the TC was seen more commonly in men than in women. Another cadaveric study demonstrated that the TC was found at $13 \%$ in the Nigerian population [2]. Researchers have found the prevalence of the TC at $65.5 \%$ in the Chinese population (Table 5) [21]. Our study was completed among the Turkish population and indicated the highest percentage among populations. These studies point out that the TC could be present in various percentages in different populations.

Besides that, being aware of the TC is also important in forensic medicine. A study showed that the TC would be misdiagnosed with the fracture of the superior horn of thyroid cartilage $[9,10]$.

Additionally, our results can be regarded as the first report about detailed investigation of relation between age and ossification degree and density of the ossified triticeous cartilage.

\section{CONCLUSIONS}

In conclusion, cadaveric dissection is indispensable for anatomy education and studies. On the other hand, with the development of detailed monitoring systems, cooperation between anatomy and clinical sciences is improved. Due to our aim having been to raise clinical attention, we completed the study with using routine CTA procedures. Besides that, the main limitation of this study was the insufficient number of cadaveric specimens.

The TC is seen commonly with different appearances in a large adult population. Other prevalence studies in various populations showed that the TC could be found in different percentages. Further studies are required in different population to determine the prevalence of the TC in worldwide. By using CTA images, more accurate and detailed data about the TC could be obtained. According to our results, the prevalence of the TC is $68.1 \%$, with variable morphological properties. Because of those differences of the TC's prevalence and morphological features, clinicians' awareness of TC is crucial for avoiding misdiagnosis of the TC with other pathological cases. 


\section{Acknowledgements}

We thank Professor Mutlu Hayran, MD, PhD, for his contribution in statistical analysis.

\section{REFERENCES}

1. Ahmad M, Madden R, Perez L. Triticeous cartilage: prevalence on panoramic radiographs and diagnostic criteria. Oral Surg Oral Med Oral Pathol Oral Radiol Endod. 2005; 99(2): 225-230, doi: 10.1016/j.tripleo.2004.06.069, indexed in Pubmed: 15660097.

2. Ajmani ML. A metrical study of the laryngeal skeleton in adult Nigerians. J Anat. 1990; 171: 187-191, indexed in Pubmed: 2081705.

3. Alqahtani E, Marrero DE, Champion WL, et al. Triticeous cartilage CT imaging characteristics, prevalence, extent, and distribution of ossification. Otolaryngol Head Neck Surg. 2016; 154(1): 131-137, doi: 10.1177/0194599815615350, indexed in Pubmed: 26556461.

4. Alsarraf R, Mathison S, Futran N. Symptomatic presentation of an enlarged, ossified triticeal cartilage. Am J Otolaryngol. 1998; 19(5): 339-341, indexed in Pubmed: 9758185.

5. Carter LC. Discrimination between calcified triticeous cartilage and calcified carotid atheroma on panoramic radiography. Oral Surg Oral Med Oral Pathol Oral Radiol Endod. 2000; 90(1): 108-110, doi: 10.1067/moe.2000.106297, indexed in Pubmed: 10884645.

6. Claassen $H$, Schicht $M$, Sel S, et al. Special pattern of endochondral ossification in human laryngeal cartilages: $\mathrm{X}$-ray and light-microscopic studies on thyroid cartilage. Clin Anat. 2014; 27(3): 423-430, doi: 10.1002/ca.22309, indexed in Pubmed: 24496984.

7. Cohen SN, Friedlander AH, Jolly DA, et al. Carotid calcification on panoramic radiographs: an important marker for vascular risk. Oral Surg Oral Med Oral Pathol Oral Radiol Endod. 2002; 94(4): 510-514, indexed in Pubmed: 12374929.

8. Dang-Tran KD, Dedouit F, Joffre F, et al. Thyroid cartilage ossification and multislice computed tomography examination: a useful tool for age assessment? J Forensic Sci. 2010; 55(3): 677-683, doi: 10.1111/j.15564029.2010.01318.x, indexed in Pubmed: 20345806.

9. Di Nunno N, Lombardo S, Costantinides F, et al. Anomalies and alterations of the hyoid-larynx complex in forensic radiographic studies. Am J Forensic Med Pathol. 2004; 25(1): 14-19, doi: 10.1097/01.paf.0000113931.49721. e4, indexed in Pubmed: 15075682.

10. Fais $P$, Giraudo C, Viero A, et al. Micro computed tomography features of laryngeal fractures in a case of fatal manual strangulation. Leg Med (Tokyo). 2016; 18: 85-89, doi: 10.1016/j.legalmed.2016.01.001, indexed in Pubmed: 26832384.
11. Garvin HM. Ossification of laryngeal structures as indicators of age. J Forensic Sci. 2008; 53(5): 1023-1027, doi: 10.1111/j.1556-4029.2008.00793.x, indexed in Pubmed: 18624888.

12. Guimarães Henriques JC, Kreich EM, Helena Baldani M, et al. Panoramic radiography in the diagnosis of carotid artery atheromas and the associated risk factors. Open Dent J. 2011; 5: 79-83, doi: 10.2174/187421060110501 0079, indexed in Pubmed: 21760860.

13. Kamikawa RS, Pereira MF, Fernandes A, et al. Study of the localization of radiopacities similar to calcified carotid atheroma by means of panoramic radiography. Oral Surg Oral Med Oral Pathol Oral Radiol Endod. 2006; 101(3): 374-378, doi: 10.1016/j.tripleo.2005.03.030, indexed in Pubmed: 16504872.

14. Mohini M. Joshi SDJ, Sharda S Joshi. Prevalence and Variations of Cartilago Triticea. Int J Anat Res. 2014; 2(3): 474-477.

15. Pornprasertsuk-Damrongsri S, Thanakun S. Carotid artery calcification detected on panoramic radiographs in a group of Thai population. Oral Surg Oral Med Oral Pathol Oral Radiol Endod. 2006; 101(1): 110-115, doi: 10.1016/j. tripleo.2005.04.002, indexed in Pubmed: 16360615.

16. Sakamoto Y. Gross anatomical observations of attachments of the middle pharyngeal constrictor. Clin Anat. 2014; 27(4): 603-609, doi: 10.1002/ca.22344, indexed in Pubmed: 24343865.

17. Saternus KS, Maxeiner H, Kernbach-Wighton G, et al. Traumatology of the superior thyroid horns in suicidal hanging: an injury analysis. Leg Med (Tokyo). 2013; 15(3): 134-139, doi: 10.1016/j.legalmed.2012.10.008, indexed in Pubmed: 23266404.

18. Standring S. Gray's anatomy: The Anatomical Basis of Clinical Practice. 41 Edn. New York: Elsevier Limited. 2016; 18: 1562.

19. Tamura $T$, Inui $M$, Nakase $M$, et al. Clinicostatistical study of carotid calcification on panoramic radiographs. Oral Dis. 2005; 11(5): 314-317, doi: 10.1111/j.16010825.2005.01125.x, indexed in Pubmed: 16120119.

20. Türkmen S, Cansu A, Türedi S, et al. Age-dependent structural and radiological changes in the larynx. Clin Radiol. 2012; 67(11): e22-e26, doi: 10.1016/j.crad.2012.07.006, indexed in Pubmed: 22938793.

21. Watanabe H, Kurihara K, Murai T. A morphometrical study of laryngeal cartilages. Med Sci Law. 1982; 22(4): 255-260, doi: 10.1177/002580248202200406, indexed in Pubmed: 7144460.

22. Wilson I, Stevens J, Gnananandan J, et al. Triticeal cartilage: the forgotten cartilage. Surg Radiol Anat. 2017; 39(10): 1135-1141, doi: 10.1007/s00276-017-1841-z, indexed in Pubmed: 28314939. 